\title{
EFFECTIVENESS OF DIFFUSION WEIGHTED MRI DERIVED METRICS IN DIFFERENTIATION OF PEDIATRIC POSTERIOR FOSSA TUMORS
}

\author{
Eman A. F. Darwish, Aliaa S. Sheha and Ahmed S. Abdelrahman
}

Radiology Department, Faculty of Medicine, Ain Shams University,Cairo, Egypt

\section{Corresponding author:} Eman A.F. Darwish

Mobile: 01063684444 E mail:

emand_9@yahoo.com

Received: $11 / 3 / 2019$

Accepted: 2/4/2019

\begin{abstract}
:
Background: The value of diffusion weighted imaging (DWI) in differentiation of pediatric posterior fossa tumors (PFTS)has been established previously, however most studies were limited only to the most common types of PFTs and their results were contradictory..
\end{abstract}

Aim of work: The aim of this study was to investigate the effectiveness of DWI in differentiation of PFTs taking care to include the less frequent types, and to clarify previously reported debatable findings.

Patients and methods: We retrospectively analyzed 13 embryonal tumors (EMBTs), 4 ependymomas (EPNs), 8 low grade gliomas (LGGs),2 mixed neuronal glial tumors (MNGTs), and 5 diffuse intrinsic pontine gliomas (DIPGs). For all tumors, absolute mean $A D C$ of the non necrotic enhancing portions of the tumor and $A D C$ ratios of the solid enhancing region of the tumor to the ADC of normal appearing cerebellum were generated. The ADC metrics of the various groups (designated according to histological subtype and WHO grade) were compared using the kruskal - wallis and student-ttests. Receiver operating characteristic (ROC) curve analysis was used to generate cutoff absolute ADC values and $A D C$ ratios for differentiation of different types of PFTs

Results: Mean $A D C$ values and $A D C$ ratios were significantly lower in high than in low grade tumors $(P<0.0001)$. Kruskal-wallis test showed statistically significant difference in the ADC metrics among the tumor groups $(P=0.000)$. Mean $A D C$ values were significantly higher in $L G G s(1.71+/-0.13)$ than in ependymomas $(1.13+/-0.13)$ and both were significantly higher than EMBTs (0.69+/-0.09). No overlap was seen in the range of $A D C$ values and ratios of EMBTs, EPNs, and LGGs. DIPG mean ADC values and ratios overlapped widely with EPNs from which they could not be differentiated using ADC metrics. DIPGs could be distinguished from EMBTs using a cutoff ADC ratio of $>1.108 \times 10-3$ (100\% sensitivity and $92.3 \%$ specificity) but not with mean $A D C$ values and from $L G G s$ using a cutoff mean ADC value of $\leq 1.333$ (100\% sensitivity and specificity). No statistically significant difference $(P>0.05)$ was seen between the mean ADC values of MNGTs (1.5+/-0.02) and LGGs $(1.71+/-0.13)$ with wide overlap in their range of $A D C$ values and ratios.

Conclusion: Apart from a few exceptions, absolute ADC values and ratios go a long way in enabling pre-operative differentiation of different types of PFTs. 
Keywords: diffusion weighted imaging, posterior fossa tumors, apparent diffusion coefficient, medulloblastomas

\section{INTRODUCTION:}

Tumors of the central nervous system (CNS) are the most frequently encountered type of solid tumors in children, over half of which are located within the posterior fossa ${ }^{[1]}$. High and low grade gliomas, embryonal tumors namely medulloblastomas (MB) and atypical teratoid rhabdoid tumors (ATRT), in addition to ependymomas (EP) constitute the most prevalent pediatric posterior fossa tumors (PFT). Mixed neuronal-glial tumors, hemangioblastomas and fourth ventricular choroid plexus tumors are less commonly seen $^{[2]}$. Precise preoperative characterization of the tumor type is critical as different tumors demand different surgical approaches and vary significantly in their prognosis. Conventional magnetic resonance imaging (MRI) has long been considered the gold standard in the detection of the tumor and determination of its extent and effects, however conventional sequences offer inadequate information regarding tumor type and $\operatorname{grade}^{[3]}$.

Diffusion weighted imaging (DWI) is an MR-based technique that enables analysis of the random microscopic motion of water molecules within biological tissues. The automatically generated apparent diffusion coefficient (ADC) map allows quantitative measurement of water diffusion within each voxel. Tumoral signal intensity on DWI and ADC values are dependent primarily on tumor cellularity, where more cellular tumors demonstrate reduced extracellular space which hinders the diffusion of water molecules resulting in lower ADC values and vice versa ${ }^{[3]}$. Given that PFTs differ significantly in terms of cellularity, it is not surprising that several previous studies have elucidated the valuable role of DWI in differentiation of PFT histological types and grades. Nevertheless, the sensitivity and specificity of the various ADC values in differentiation of the different tumor types varied among the different studies and significant overlap in the ADC values of the various PFTs was reported. Furthermore, the majority of these studies failed to incorporate the rarer types of PFTs, preferring to concentrate only on juvenile pilocytic astrocytomas (JPA), MBs, and ependymomas $^{[2-5]}$.

\section{AIM OF THE WORK:}

The aim of this work was to investigate the effectiveness of DWI and ADC values in differentiation of the various PFT types and grades, taking care to include a wider range of PFT types, and to clear up the previous controversial findings.

\section{PATIENTS AND METHODS:}

This study was approved by our department's research ethics board.

\section{Patients:}

We retrospectively reviewed our PACS system for pediatric patients, younger than 18 years, who fulfilled our predefined inclusion criteria over a time period extending from January 2017 to December 2017. The inclusion criteria for this study were:

The presence of a histopathologically provenintraxial posterior fossa tumor or the presence of a brainstem tumor with MRI features consistent with the diagnosis of DIPG as described in the literature $^{[6-8]}$. Imaging features suggestive of DIPGs include:

o Mass epicentered on the pons

o involves more than $50-75 \%$ of the cross sectional area of the pons which 
appears expanded and diffusely infiltrated

o hypointense on T1-weighted image/ hyperintense on T2-weighted image

o no to mild contrast enhancement

o may engulf the basilar arteryanteriorly

N.B. histopathological confirmation was waived for tumors with features consistent with DIPGs as these tumors are not routinely biopsied at our institution.

The presence of a complete pretreatment MR study including T1, and T2-weighted images, fluid-attenuated inversion recovery (FLAIR), postcontrast T1-weighted and DWI images.

$>$ Solid portions of the tumor must be large enough to allow adequate region of interest (ROI) analysis.
Excluded from the study were patients with entirely cystic or extraaxialtumors and patients who received treatment for the tumor prior to the MR performed at our institution.

A total of 32 patients met our inclusion criteria and were enrolled in our study.

\section{Magnetic resonance imaging:}

All examinations were performed on Philips Achieva 1.5 Tesla MR and consisted of our standard imaging protocol for brain tumors which include pre and post contrast conventional MR sequences in addition to the diffusion weighted sequences which were acquired with effective $b$-values of 0 and $1,000 \mathrm{~s} / \mathrm{mm} 2$ using a single-shot echoplanar sequence. ADC maps were automatically generated. The various sequences and their acquisition parameters are listed in table 1

Table 1: Imaging parameters of the various MR sequences

$B W$ - bandwidth, deg- degrees, FA - flip angle, FLAIR- fluid attenuated inversion recovery, FOV-

\begin{tabular}{|c|c|c|c|c|c|c|c|c|}
\hline Sequences & $\begin{array}{l}\text { Imaging } \\
\text { plane }\end{array}$ & $\begin{array}{l}\text { TR/TE } \\
(\mathrm{ms})\end{array}$ & $\begin{array}{l}\text { Acquisition } \\
\text { time }\end{array}$ & $\begin{array}{l}\text { Voxel size } \\
(\mathrm{mm})\end{array}$ & $\begin{array}{l}\text { FOV } \\
(\mathbf{m m})\end{array}$ & Matrix & $\begin{array}{l}\text { Slice } \\
\text { thickness } \\
(\mathbf{m m})\end{array}$ & $\begin{array}{l}\text { FA/BW } \\
\text { (Deg/Hz) }\end{array}$ \\
\hline SE T1 & Axial & $597 / 15$ & $1 \mathrm{~min}, 20 \mathrm{sec}$ & $1.1 / 1.35 / 5$ & $200 \times 230$ & $200 \times 133$ & 5 & $69 / 108.7$ \\
\hline TSE T2 & Axial & $4845 / 110$ & $38 \mathrm{sec}$ & $0.9 / 1.12 / 5$ & $200 \times 230$ & $244 \times 147$ & 5 & $90 / 213.5$ \\
\hline FLAIR & Axial & $11000 / 130$ & $2 \mathrm{~min}, 45 \mathrm{sec}$ & $0.9 / 1.19 / 5$ & $220 \times 230$ & $228 \times 120$ & 5 & $100 / 328.5$ \\
\hline $\begin{array}{l}\text { Post } \\
\text { contrast T1 }\end{array}$ & Axial & $514 / 15$ & $2 \mathrm{~min}, 31 \mathrm{sec}$ & $1.1 / 1.38 / 5$ & $200 \times 230$ & $200 \times 130$ & 5 & $68 / 108.7$ \\
\hline $\begin{array}{l}\text { Post } \\
\text { contrast T1 }\end{array}$ & Coronal & $154 / 1.83$ & $51 \mathrm{sec}$ & $1 / 1.2 / 5.5$ & $230 \times 200$ & $32 \times 164$ & 5.5 & $80 / 189.7$ \\
\hline $\begin{array}{l}\text { Post } \\
\text { contrast T1 }\end{array}$ & Sagittal & $152 / 1.9$ & $31 \mathrm{sec}$ & $0.9 / 1.9 / 5.5$ & $230 \times 230$ & $256 \times 205$ & 5.5 & $80 / 171.9$ \\
\hline DWI & Axial & $4124 / 118$ & $1 \mathrm{~min}, 26 \mathrm{sec}$ & $1.5 / 2.21 / 5$ & $190 \times 232$ & $128 \times 105$ & 5 & $90 / 17.7$ \\
\hline
\end{tabular}

field of view, $\mathrm{Hz}$ - hertz, $\mathrm{mm}$ - millimeter, $\mathrm{ms}$ - millisecond, sec-second, $S E$ - spin echo, $T E$ - echo time, $T R$ - repetition time, TSE- turbo spin echo

\section{Image analysis:}

Analysis of the images was carried out on our workstation by two experienced neuroradiologists blinded to the results of histopathology and decisions were reached by consensus. Predominant signal intensity of the tumor was assessed qualitatively on ADC map and was designated as hyperintense, isointense, or hypointense / iso-hypointense / isointense / iso-hyperintense / hyperintense relative to the adjacent normal brain parenchyma. An area of restricted diffusion was recognized by high signal intensity on the b-1000 image and low signal intensity on the corresponding ADC map. Conventional pre and post contrast MR sequences were 
inspected to localize the tumor and identify the solid, non necrotic, non hemorrhagic, non calcific enhancing portion of the tumor. Quantitative ADC measurements were obtained by drawing at least 3 regions of interest (ROIs) on the ADC map in the identified area of the tumor as described above, one on each slice to encompass the entire targeted portion. If the area to be analyzed is small, 3 ROIs were drawn within the target area on the same slice taking care to avoid overlap between the ROIs. Mean ADC values for each ROI were recorded and an average of all the values was subsequently calculated to yield amean ADC (ADCm) for each tumor. An additional ROI was then drawn in the normal cerebellar parenchymato obtain control cerebellar ADC measurements. Ratios of tumor mean ADC to cerebellar control ADC values were calculated for all tumors.

\section{Statistical analysis:}

Tumors were broadly classified into high and low grade tumors according to their WHO grade. Apart from DIPGs, which were diagnosed based on MR features, other tumors were classified into the following groups according to the results of histopathological analysis:

$>$ Low grade gliomas (LGGs)

$>$ Ependymomas (EPNs)

$>$ Mixed neuronal - glial tumors (MNGTs)

$>$ Embryonal tumors (EMBTs)

Descriptive statistics including tumor mean $\mathrm{ADC}$ and $\mathrm{ADC}$ ratios relative to the cerebellum for each of the above groups were reported as mean \pm standard deviation ( $-x \pm s d$ ), minimum-maximum, median and interquartile range (IQR),. Comparisons of the ADCm and ADC ratios between the high and low grade groups were performed using the Student-T-test. Comparisons of the $\mathrm{ADCm}$ and $\mathrm{ADC}$ ratios between the different tumor groups were performed using the Kruskal-Wallis test and Conover post hoc test was used for pair wise comparisons of the different groups.

The signal intensity of the individual tumors was assigned grades from 1 to 5 as follows:

Hypointense: grade 1

Iso to hypointense: grade 2

Isointense: grade 3

Iso to hyperintense: grade 4

Hyperintense :grade 5

The difference in the frequency of various grades of signal intensity between the different tumor groups was evaluated by the Chi-squared test. Linear regression analysis was used to evaluate the relationship between the ADC measurements and signal intensity grades

Linear regression analysis was used to evaluate the relationship between WHO grades and tumor types. Since we did not know the exact WHO grade of the individual DIPGs, they were excluded from the regression analysis.ROC analysis was performed to determine the best cutoff ADC values to differentiate between high and low grade PFT and between the different histopathological groups.

Statistical analysis was performed using 2019 Med Calcsoftware. Results were considered statistically significant if the $\mathrm{P}$ value was less than 0.05 .

\section{RESULTS:}

32 children (20 males and 12 females) met our inclusion criteria. The mean age of our patients was6.25 3.26 (range 1-15 years). Histopathological evaluation was present for 27 PFT in our study and revealed the presence of $13(40.6 \%)$ embryonal tumors (9of which were MBs while 4 were ATRTs), 5 (15.6\%), 4 (12.5\%) ependymomas, $8(25 \%)$ LGGs (6 of which were juvenile pilocytic astrocytomas while 2 were pilomyxoidastrocytomas) and 2 (6.25\%) 
MNGTs (both of which were gangliogliomas). 5 (15.6\%) DIPGs were enrolled in this study and the diagnosis was made based on conventional MRI features as described before. All EMBTs were WHO grade IV, 2 of the ependymomas were WHO grade III, two of the ependymomas, both of the PMAs were WHO grade II, while all of the JPAs and GGs were WHO grade I. WHO grade I and II tumors are low grade tumors, whereas WHO grades III and IV tumors are high grade tumors. Since histopathological analysis was not available for DIPGs they could not be assigned a definite WHO grade, nonetheless they were considered to be high grade tumors in concordance with information Table 2: Summary of ADC values and ADC ratios of tumors to cerebellum for high and low grade tumors

\begin{tabular}{|l|l|l|l|}
\hline ADC & $\begin{array}{l}\text { High grade tumors }(\mathrm{n}=20, \\
\text { mean } \pm \mathrm{SD})\end{array}$ & $\begin{array}{l}\text { Low grade tumors }(\mathrm{n}=12, \\
\text { mean }+/ \mathrm{SD})\end{array}$ & $\mathrm{P}$ value \\
\hline Mean $\mathrm{ADC} \times 10^{-3} \mathrm{~mm}^{2} / \mathrm{s}$ & $0.7992+/-0.2305$ & $1.5925+/-0.2118$ & $\mathrm{P}<0.0001$ \\
\hline Tumor/Cerebellum ADC ratio & $1.1228+/-0.3353$ & $1.9940+/-0.5092$ & $\mathrm{P}<0.0001$ \\
\hline
\end{tabular}

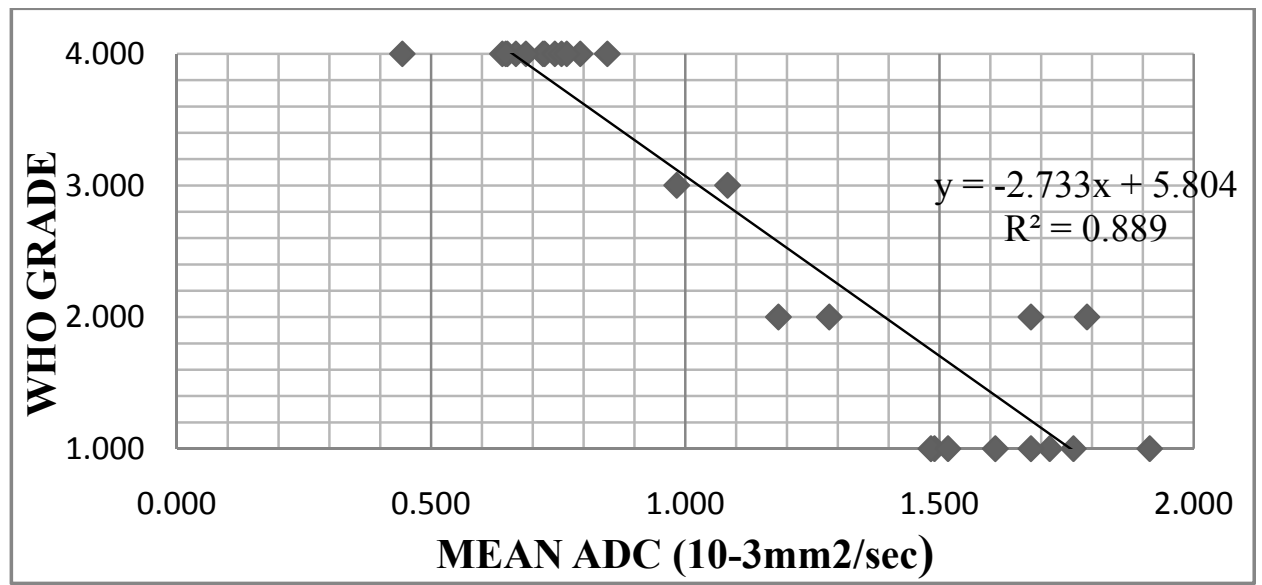

Diagram 1: Scatter diagram displaying the inverse relationship between mean ADC values and the WHO grade.

ADCm values and ratios of tumoral $\mathrm{ADCm}$ to the apparently normal cerebellum available in literature [9].Accordingly we had a total of $20(62.5 \%)$ high grade tumors and $12(37.5 \%)$ low grade tumors in this study.

Statistically significant differences existed between the ADCm values and ADC ratios of high and low grade tumors (Table 2). High grade tumors were distinguished from low grade tumors with a sensitivity of $90 \%$ and a specificity of $100 \%$ using $\mathrm{aADCm}$ of $\leq 1.083$. Linear regression analysis revealed that mean ADC values are a significant predictor of WHO grade $\left(\mathrm{R}^{2}=0.889, \mathrm{P}<0.0001\right)$ with lower ADC values associated with higher tumor grades and vice versa (Diagram 1). 
Eman A. F. Darwish, et al.,

Table 3: Summary of mean ADC values and ADC ratios of tumors to cerebellum (CB), for all tumor groups. Data are expressed in terms of median, interquartile rang (IQR),range and mean+/-standard deviation (SD).

\begin{tabular}{|l|c|c|c|c|c|c|c|c|c|}
\hline Tumor & $\mathrm{N}$ & \multicolumn{4}{|c|}{ MEAN ADC } & \multicolumn{4}{c|}{ MEAN ADC:CB } \\
\cline { 3 - 10 } & & Median & IQR & Range & Mean \pm SD & Median & IQR & Range & Mean \pm SD \\
\hline EMBT & 13 & 0.72 & $0.65-0.76$ & $0.44-0.85$ & $0.69 \pm 0.09$ & 0.91 & $0.86-1.04$ & $0.49-1.21$ & $0.93 \pm 0.17$ \\
\hline EPN & 4 & 1.13 & $1.03-1.23$ & $0.98-1.28$ & $1.13 \pm 0.13$ & 1.32 & $1.29-1.36$ & $1.27-1.39$ & $1.32 \pm 0.05$ \\
\hline DIPG & 5 & 0.92 & $0.64-1.33$ & $0.59-1.33$ & $0.97 \pm 0.36$ & 1.52 & $1.30-1.79$ & $1.17-1.85$ & $1.53 \pm 0.29$ \\
\hline LGG & 8 & 1.69 & $1.65-1.78$ & $1.49-1.91$ & $1.71 \pm 0.13$ & 2.34 & $2.09-2.44$ & $1.69-2.52$ & $2.24 \pm 0.27$ \\
\hline MNGT & 2 & 1.5 & $1.48-1.52$ & $1.48-1.52$ & $1.5 \pm 0.02$ & 1.72 & $1.13-2.29$ & $1.13-2.29$ & $1.72 \pm 0.82$ \\
\hline
\end{tabular}

Group comparisons between the mean $\mathrm{ADC}$ values of the 5 tumor groups as well as the ratios of the mean ADC to cerebellum ADC revealed a statistically significant difference between the groups as demonstrated by the kruskal-Wallis test $(\mathrm{P}=0.000)$. Conover post hoc analysis showed significant differences between the mean ADC values of EBNT group and EPN, LGG, and MNGT groups $(\mathrm{P}<0.05)$, between EPN and LGG groups $(\mathrm{P}<0.05)$, and between DIPG and LGG and MNG groups $(\mathrm{P}<0.05)$. No significant difference was seen between EMBT and DIPG groups, between the EPN and DIPG groups, between the EPN and MNGT groups and between the LGGs and MNGTs (Diagram 2). Details of the pair-wise comparisons of the different groups with regards to the ADC ratios, as demonstrated by the Conover analysis can be seen in table 4 .

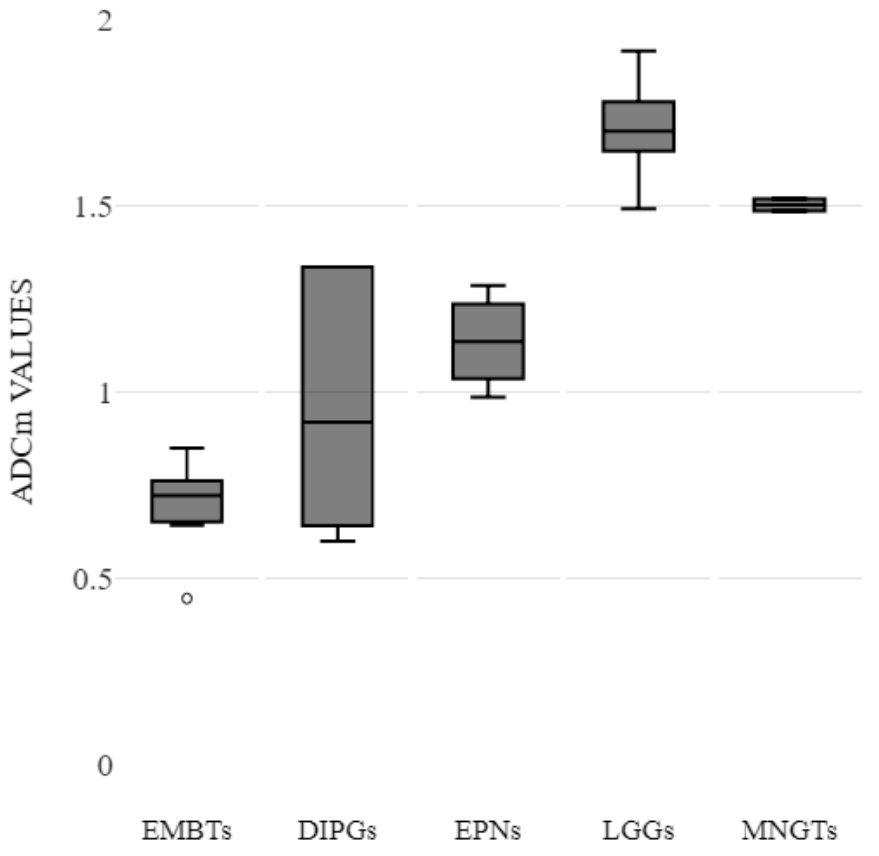

Diagram 2: Box plot for ADCm in the 5 tumor groups. Middle lines represent median values, boxes represent 25 th to 75 th percentiles, and whiskers demonstrate range. No overlap was seen in the individual ADCm values between the EMBTs, EPNs and LGGs Significant overlap was seen in ADCm values between the DIPG group and both EMBTs and EPNs as well as between LGGs and MNGTs. 
Table 4: results of pair-wise comparisons of the ratio of the tumoral mean ADC to the normal appearing cerebellum.

\begin{tabular}{|l|l|}
\hline Tumor group & Statistically significant different groups $(\mathbf{p}<\mathbf{0 . 0 5})$ \\
\hline EMBTs & EPNs,DIPGs,LGGs,MNGTs \\
\hline EPNs & EMBTs, LGGs \\
\hline DIPGs & EMBTs, LGGs \\
\hline LGGs & EMBTs, EPNs,DIPGs,MNGTs \\
\hline MNGTs & EMBTs, LGGs \\
\hline
\end{tabular}

EMBTs could be differentiated from EPNs, LGG, and MNGT using a cutoff ADCm of $\leq 0.847 \times 10^{-3} \mathrm{~mm}^{2} / \mathrm{s}$ with a sensitivity and specificity of $100 \%$. When DIPGs were added, EMBTs could be differentiated from all tumors with same cutoff value but with a sensitivity of $100 \%$ and a specificity of $89.4 \%$ due to overlap of ADCm values of DIPGs and EMBTs.

LGGs could be differentiated from EPNs using a cutoff ADCm of $>1.283 \times$ $10^{-3} \mathrm{~mm}^{2} / \mathrm{s}$ with a sensitivity and specificity of $100 \%$, from DIPGs using a cutoff ADCm value of $>1.33 \times 10^{-3} \mathrm{~mm}^{2} / \mathrm{s}$ with a sensitivity and specificity of $100 \%$ and from all tumors including MNGTs using cutoff ADCm value of $>1.48 \times 10^{-3} \mathrm{~mm}^{2} / \mathrm{s}$ with a sensitivity and specificity of $100 \%$ and $95.6 \%$ respectively. This was due to overlap in ADC values of LGGs and MNGTs.

Ependymomas could be differentiated from LGGs and MNGTs using cutoff ADCm of $\leq 1.283 \times 10^{-3} \mathrm{~mm}^{2} / \mathrm{s}$ with a sensitivity and specificity of $100 \%$ and from EMBTs using a cutoff ADCm of $>0.847 \times$ $10^{-3} \mathrm{~mm}^{2} / \mathrm{s}$ with a sensitivity and specificity of $100 \%$. DIPGs and EPNs could not be differentiated on basis of ADCm values

EMBTs could be differentiated from EPNs, and LGGs using a cutoff ADCm: CB ratio of $\leq 1.21 \times 10^{-3} \mathrm{~mm}^{2} / \mathrm{s}$ with a sensitivity and specificity of $100 \%$. When DIPGs were included, EMBTs could be differentiated from all tumor types using a cutoff $\mathrm{ADCm}$ : $\mathrm{CB}$ ratio of $\leq 1.108 \times 10^{-3} \mathrm{~mm}^{2} / \mathrm{s}$ with a sensitivity and specificity of $92.3 \%$ and
$100 \%$ respectively. EMBTs could be distinguished from DIPGs alone using the same cutoff value with an identical sensitivity and specificity.

LGGs could be differentiated from EPNs using a cutoff ADCm: $\mathrm{CB}$ of $>1.39 \times$ $10^{-3} \mathrm{~mm}^{2} / \mathrm{s}$ with a sensitivity and specificity of $100 \%$, from DIPGs alone using a cutoff ADCm: $\mathrm{CB}$ ratio of $>1.85 \times 10^{-3} \mathrm{~mm}^{2} / \mathrm{s}$ with a sensitivity and specificity of $87.5 \%$ and $100 \%$ respectively and from all tumor types using a cutoff $\mathrm{ADCm}$ : $\mathrm{CB}$ of $>1.52 \times$ $10^{-3} \mathrm{~mm}^{2} / \mathrm{s}$ with a sensitivity and specificity of $100 \%$ and $87.5 \%$. Findings can be explained by the fact that ADC ratios of LGGs overlapped with those of DIPGs and MNGTs.

EPNs could be distinguished from EMBTs and LGGs using a cutoff ADC ratios of $\leq 1.21$ and $>1.39$ respectively with a100\% sensitivity and specificity. DIPGs and EPNs could not be differentiated on basis of their ADC ratios.

The distribution of the various tumors according to signal intensity is seen in table 5.The Chi-squared test revealed a significant association between signal intensity and tumor type; $\chi 2=32.547, p=0.0011$. There was a significant positive correlation between signal intensity and both $\mathrm{ADCm}$ $\left(\mathrm{R}^{2}=0.549, \mathrm{P}<0.0001\right)$ and $\mathrm{ADCm}: \mathrm{CB}$ but the correlation between signal intensity grade and ratio of tumor ADC to cerebellar ADC was better; $\mathrm{R}^{2}=0.572, \mathrm{P}<0.0001$ (Diagram 3). 


\section{Eman A. F. Darwish, et al.,}

Table 5: distribution of individual tumors according to signal intensity

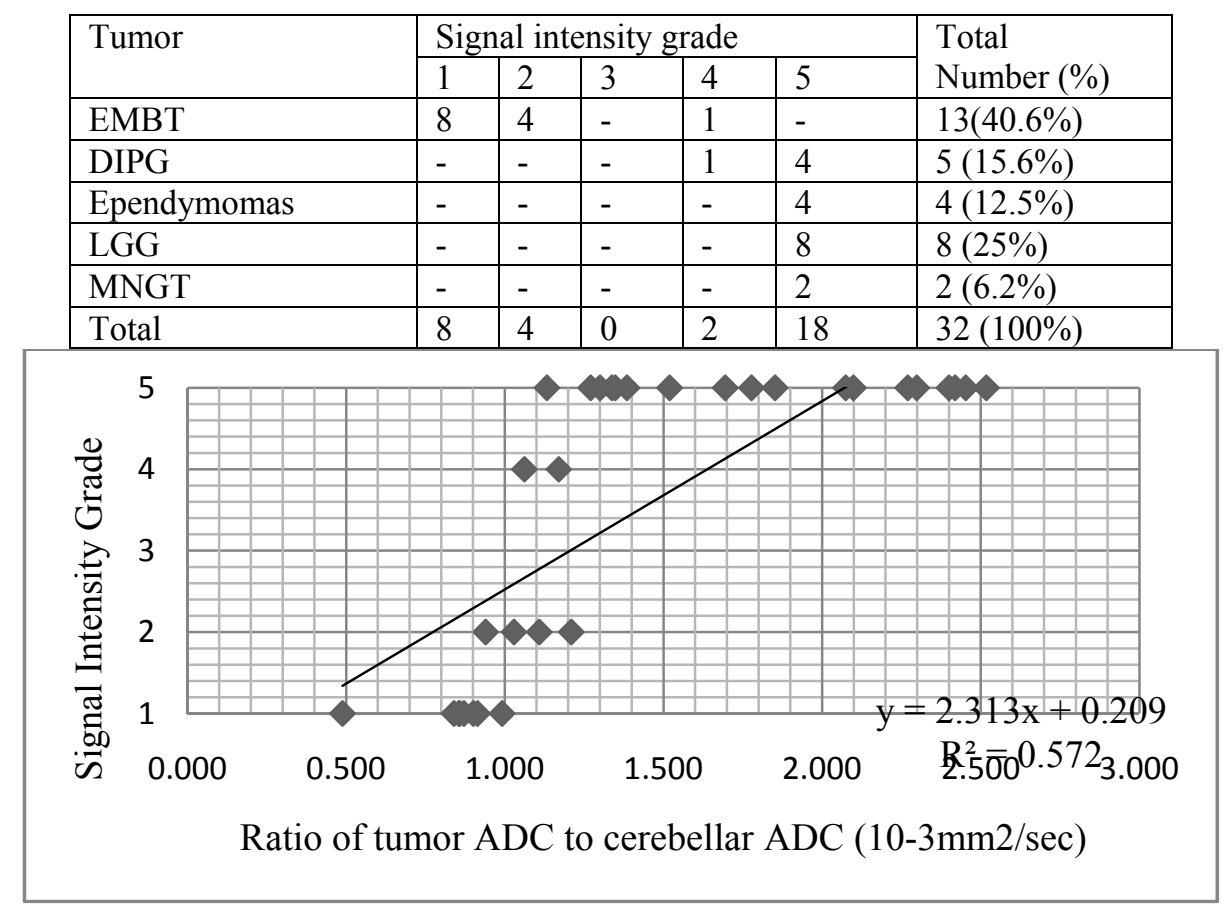

Diagram 3: Correlation between the ratio of tumor $\mathrm{ADCm}$ to the $\mathrm{ADC}$ of the normal appearing cerebellum and signal intensity grade.

Examples of the different PFTs and their qualitative and quantitative diffusion weighted characteristics can be seen in figures 1-4.
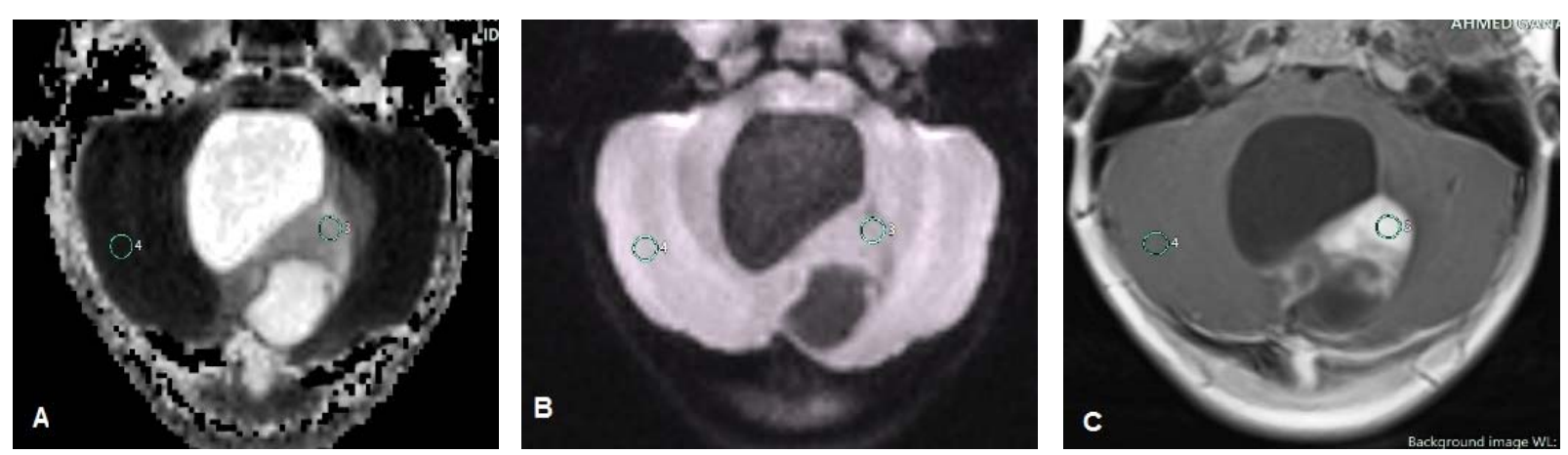

Figure 1: Eight-year-old female with histologically proven cerebellar pilocytic astrocytoma. A: Axial ADC map reveals a midline infra tentorial cystic tumor with peripherally located solid mural nodule. The tumor displays a hyper intense signal compared to the surrounding cerebellum. Mean ADC was $=$ $1.763 \times 10^{-3} \mathrm{~mm}^{2} / \mathrm{s}$. and the ADC ratio to normal cerebellum was $2.075 \times 10^{-3} \mathrm{~mm}^{2} / \mathrm{s}$ consistent with lack of diffusion restriction. B: Axial DWI confirmed the absence of diffusion restriction. C: Axial $\mathrm{T} 1 \mathrm{~W}$ post contrast image reveals significant enhancement of the mural nodule. 

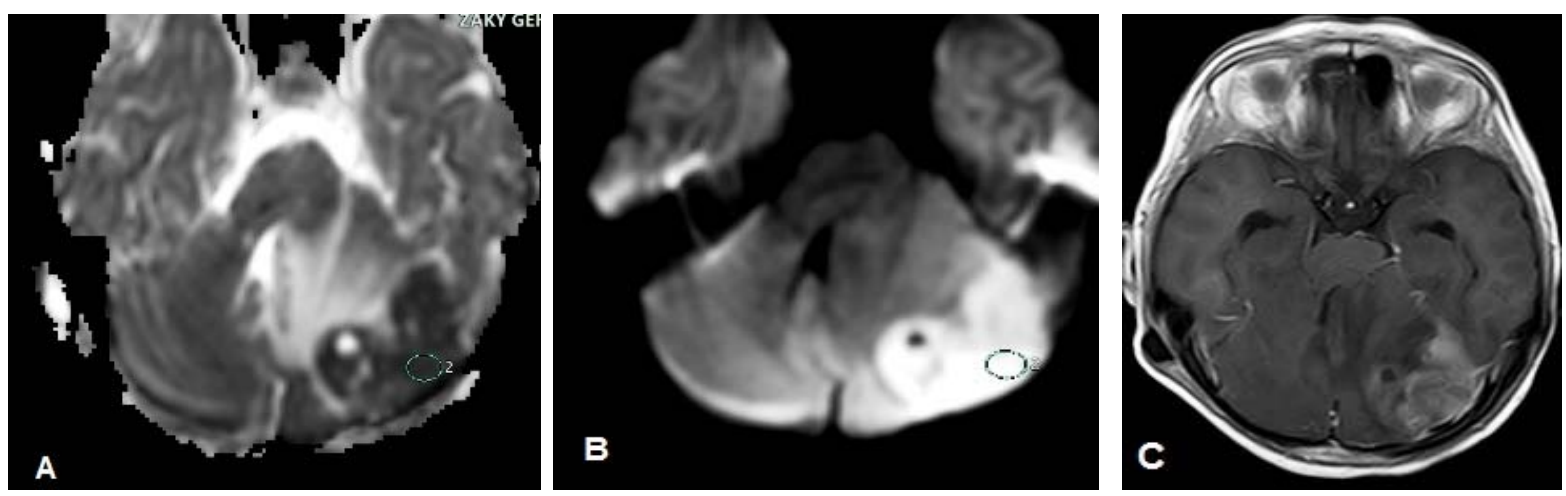

Figure 2: 5-year-old female with histologically proven cerebellar hemispheric medulloblastoma. A: Axial ADC map reveals a left cerebellar hemisphere solid tumor with surrounding vasogenic edema. The tumor displays a hypointense signal compared to the surrounding cerebellum. Mean ADC was value $=0.64 \times 10^{-3} \mathrm{~mm}^{2} / \mathrm{s}$. and the ADC ratio to normal cerebellum was $0.914 \times 10^{-3} \mathrm{~mm}^{2} / \mathrm{s}$ consistent with the presence of true diffusion restriction. B: Axial DWI confirmed the presence of diffusion restriction. C: Axial T1W post contrast image reveals significant enhancement of the tumor
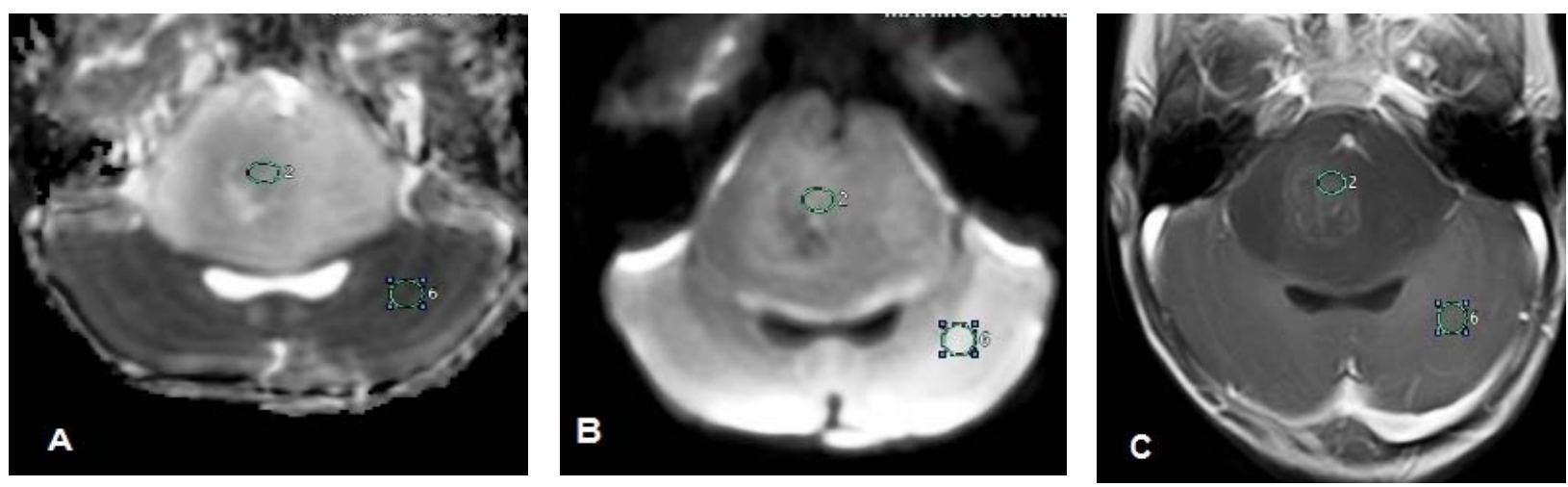

Figure 3: 3-year-old female with a diffuse intrinsic pontine glioma. A: Axial ADC map reveals a hugely expanded pons which appears to be diffusely infiltrated with a lesion of a hyperintese signal compared to the adjacent cerebellum. The fourth ventricle is compressed posteriorly and the basilar artery is engulfed anteriorly. Mean ADC was $=1.333 \times 10^{-3} \mathrm{~mm}^{2} / \mathrm{s}$. and the ADC ratio to normal cerebellum was $1.852 \times 10^{-3} \mathrm{~mm}^{2} / \mathrm{s}$ consistent with the absence of diffusion restriction. B: Axial DWI confirms the absence of diffusion restriction. C: Axial T1W post contrast image reveals the presence of a localized are of mild heterogeneous enhancement within the otherwise non enhancing tumor.
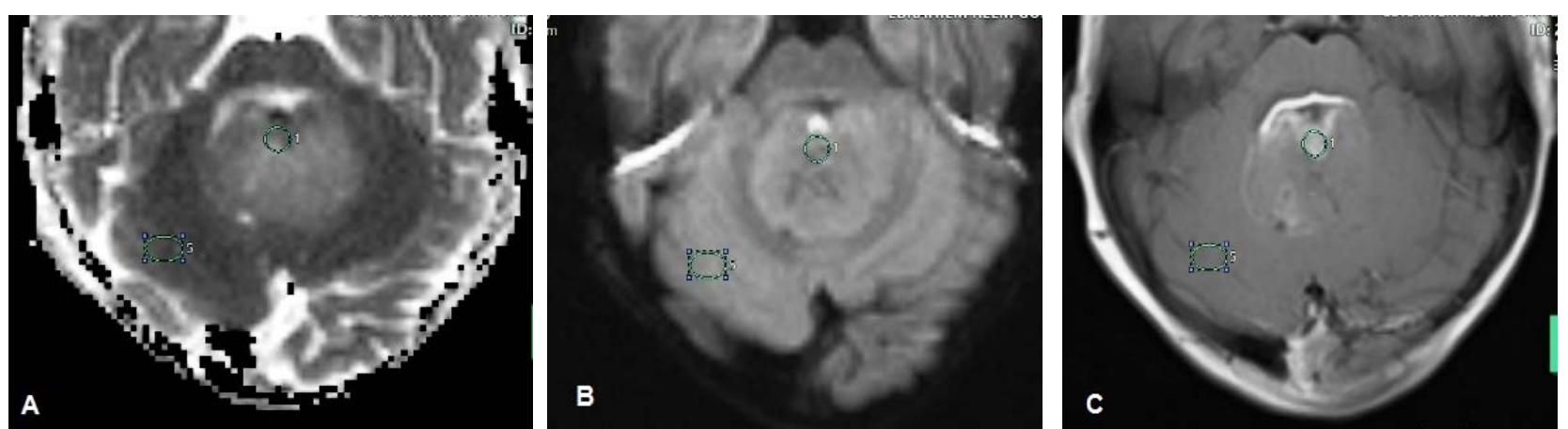

Figure 4: 4-year-old female with a histologically proven anaplastic ependymoma A: Axial ADC map reveals large, midline, solid mass centered on the $4^{\text {th }}$ ventricle. It displays a hyperintense signal compared to the adjacent cerebellum. Mean $\mathrm{ADC}$ was $=1.083 \times 10^{-3} \mathrm{~mm}^{2} / \mathrm{s}$. and the ADC ratio to normal cerebellum was $1.337 \times 10^{-3} \mathrm{~mm}^{2} / \mathrm{s}$ consistent with the absence of diffusion restriction. B: Axial DWI confirms the absence of diffusion restriction. C: Axial T1W post contrast image reveals mild to moderate patchy enhancement of the tumor. 


\section{DISCUSSION:}

DWI is an MR sequence that creates images where contrast between tissues is determined by the motion characteristics of water molecules within different tissues. The ease with which water molecules diffuse through different tissues depends on a number of factors, the most important of which is the microstructure of tissues. Diffusion of water molecules is hindered in highly cellular tumors with a high nuclearto-cytoplasmic ratio and small extracellular space, which thus appear restricted on DWI and demonstrate low ADC values on the corresponding ADC map. On the other hand tumors with low cellularity and abundant extracellular space typically show increased diffusivity and high ADC values ${ }^{[4,10]}$.

Increased cellularity is a frequent feature of high grade tumors, while low grade tumors are typically loosely packed with large extracellular spaces. In a clear reflection of these histological properties, our study demonstrated that absolute ADC values and ratios in high grade tumors were significantly lower than those of low grade tumors with a strong negative correlation between WHO grade and ADC values. Our findings were consistent with several previous studies ${ }^{[2,4,10,11-13]}$. Alternatively Zonari et al. and Catalaa et al. reported that their ADC measurements failed to distinguish between tumor grades [14, 15]. Their findings may be attributed to their different methodology and study cohort which included only glial tumors and failed to incorporate other histological subtypes, unlike this study and aforementioned studies.

Our study revealed statistically significant differences between absolute $\mathrm{ADC}$ values and ratios of tumor $\mathrm{ADC}$ to normal brain (cerebellum) of EBNTs, ependymomas, and LGGs with EBNTs displaying the lowest values, LGGs displaying the highest values, while ependymomas consistently demonstrated ADC measurements that lied between those of EBNTs and LGGs. This coincides with many older studies who have reported similar findings ${ }^{[2,3,4,5,16-19,20]}$. These consistent findings are easily explained by their individual microscopic features. MBs and ATRTs which constituted our embryonal tumors group, and are often indistinguishable on imaging, are highly cellular tumors, with tightly packed cells, high nuclear-to-cytoplasmic ratio and little extracellular matrix. The absolute ADC values and $\mathrm{ADC}$ ratios for EMBTs group in this study were $0.69 \pm 0.09 \times 10^{-3} \mathrm{~mm} 2 /$ sand $0.93 \pm 0.17 \times 10^{-3} \mathrm{~mm} 2 / \mathrm{s}$ respectively which were comparable to ADC values and ratios reported by Gemi et al., Gauvain et al, and Zitouni et al., but were higher than the absolute ADC value of $0.58 \pm 0.09 \times$ $10^{-3} \mathrm{~mm} 2 /$ sand ADC ratio of $0.78 \pm$ $0.12 \times 10^{-3} \mathrm{~mm} 2 / \mathrm{s}$, measured by Poretti et $\mathrm{al}^{[2,4,18,19]}$. This variability may be due to a difference in the histological subtypes of medullobastomas included in each study. We did not perform a correlation between the subtypes of medulloblastomas and ADC measurements, yet at least one previous study found that the more aggressive classic variant of medulloblastoma had lower ADC values than the less aggressive desmoplastic/nodular subtype, a finding supported by Jaremko et al who described the absence of diffusion restriction in a single desmoplastic medulloblastoma ${ }^{[5,21]}$. Nevertheless, Koral et al and FruehwaldPallamaret al failed to demonstrate a difference in the ADC metrics of medulloblastoma subtypes ${ }^{[22,23]}$.

The increased diffusivity of low grade gliomas which were represented by pilocytic astrocytomas and pilomyxoidastrocytomas in this study, is likely a reflection of the abundance of poorly cellular vacuolated areas in the former and large areas of myxoid matrix in the latter. Our ADC value of $1.71 \pm 0.1 \times 10^{-3} \mathrm{~mm} 2 / \mathrm{s} 3$ and ratio of 
$2.24 \pm 0.27 \times 10^{-3} \mathrm{~mm} 2 / \mathrm{s}$ for the LGGs group were comparable to the ADC value of $1.632 \times 10^{-3} \mathrm{~mm} 2 / \mathrm{s}$ and ratio of $2.29 \times$ $10^{-3} \mathrm{~mm} 2 / \mathrm{s}$ demonstrated by Gemi et al but were higher than the ADC values and ratios observed by multiple older studies ${ }^{[2,4,19,20]}$. This variation could partly stem from the fact that JPAs often vary in the degree of diffusivity of water molecules within their solid nodules. Although not encountered in our study, a previous study documented the presence of restricted diffusion in at least a single case of $\mathrm{JPA}^{[5]}$.

Ependymomas which are characterized by the presence of perivascular pseudorosettes and ependymal rosettes havean intermediate cellularity between EMBTs and LGGs [3,19]. ADC values and ratios for ependymomas ranged from 0.98 to $1.28 \times$ $10^{-3} \mathrm{~mm}^{2} / \mathrm{s}$ and from 1.27 to $1.39 \times$ $10^{-3} \mathrm{~mm}^{2} / \mathrm{s}$ respectively in this study, yet other studies found that ADC values and ratios of ependymomas span a relatively wide range with values as low and high as those of MBs and PAs respectively recorded [18-20]. This difference can be explained by the variation in the sample sizes of ependymomas assessed in the various studies and the fact that several histological variants of ependymomas exist, some of which show increased cellular packing. Additionally anaplastic grade III ependymomas are more cellular than their more benign WHO grade II counterparts ${ }^{[3]}$.

DIPGs, more recently known as diffuse midline gliomas according to the 2016 WHO classification of brain tumors,are WHO grade III and IV with a tiny subset of WHO grade II gliomas that display a distinctive genetic mutation ${ }^{[6-8,24]}$. Accordingly, it is not surprising that our study revealed that the ADC values and ratios of DIPGs covered a wide range from 0.590 to $1.327 \times 10^{-3} \mathrm{~mm}^{2} / \mathrm{s}$ and from 1.17 to $1.85 \times 10^{-3} \mathrm{~mm}^{2} / \mathrm{s}$ respectively, overlapping significantly with those of ependymomas and, more importantly, EBNTs which are the most common brainstem tumors to mimic DIPGs on imaging. Moreover, one study recently found that DIPGs demonstrated distinct intralesional heterogeneity as regards ADC measurements which may further explain the wide range of $\mathrm{ADC}$ values reported for $\mathrm{DIPG}^{[7]}$.

Similar to Poretti et al, no overlap of the absolute ADC measurements and ADC ratios between the main PFT groups namely EMBTs, ependymomas, and LGGs, was found $^{[2]}$. We could distinguish the three major tumor types with $100 \%$ sensitivity and specificity using the specific threshold ADC values and ratios. Comparable cutoff $\mathrm{ADC}$ values and ratios were reported by many studies but with varying sensitivities and specificities due to overlap in the ADC metrics between different groups ${ }^{[3,5,16-20]}$. We attribute the lack of overlap in this study partly to our relatively small sample size, as well as to our methodology which involved strict placement of the ROIs within enhancing, non necrotic, non edematous, non hemorrhagic and uncalcified portions of the tumors after careful inspection of all MR sequences.

The sensitivities and specificities of cutoff ADC values and ratio for differentiation of all tumor groups including DIPGs and MNGTs were lower than those generated for the three main PFT groups due to the significant overlap that existed in the ADC values and ratios of DIPGs and EMBTs, DIPGs and ependymomas and MNGTs and LGGs. Almost all previous studies included only the most common PFTs and so we could not verify our findings as regards DIPGs and MNGTs. However, the absolute ADC value and ratio for the single GBM that was included in the cohort studied by Poretti et al., overlapped with those of medulloblastomas ${ }^{[2]}$. Since DIPGs are essentially anaplastic astrocytomas or GBMs, this observation coincides with our findings. Although the significant overlap that exists between 
DIPGs and other tumor types may seem problematic, it is important to remember that from practical standpoint ADC metrics are not used alone in differentiation of the various tumor types, rather they are used in conjunction with conventional MRI characteristics which go a long way in narrowing down diagnostic choices. With that in mind, failing to differentiate DIPGs from ependymomas using ADC measurements becomes less important, as they can be distinguished on basis of location and other conventional features. The main imaging differential diagnoses of DIPGs are low grade gliomas and embryonal tumors of the brain stem. While our results indicate that absolute ADC values cannot be relied upon in differentiation of DIPGs and EMBTs, EMBTs could be differentiated from DIPGs with a specificity of $100 \%$ and a sensitivity of $92.3 \%$ using a cutoff ADC ratio of $\leq 1.108 \times 10^{-3} \mathrm{~mm}^{2} / \mathrm{s}$. This is due to the fact that all of the DIPGs in this study failed to demonstrate diffusion restriction which means that even though their absolute ADC values may be as low as EMBTs, their ratios are always higher. DIPGs could be differentiated from LGGs using a cutoff absolute ADC value of $\leq 1.33 \times 10^{-3} \mathrm{~mm}^{2} / \mathrm{s}$ with $100 \%$ sensitivity and specificity. Nevertheless, care must be taken as ADC measurements as high as $1.5 \times 10^{-3} \mathrm{~mm}^{2} / \mathrm{s}$ were recorded for some DIPGs ${ }^{[8]}$. Additionally Gauvain et al. assessed ependymomas and high grade gliomas together and found that their ADC ratios overlapped with LGGs ${ }^{[4]}$. Further studies with a larger group of brain stem tumors of different histologies are necessary before definite conclusions can be made.

Two cases of gangliogliomas constituted our MNGT group. GGs are rare tumors that can occur anywhere in the CNS. Most commonly they are WHO grade I lesions as was encountered in this study ${ }^{[25]}$. Thus, it is not surprising that their absolute ADC values and ratios closely overlapped with those of LGGs and could be differentiated from EBNTs, DIPGs and ependymomas using cutoff ADC measures similar to those of LGGs.

We chose to investigate the utility of absolute ADC values as well as ADC ratios in differentiation of PFTs in this study, yet in mainstream clinical practice the choice of which parameter to use remains debatable. While ADC values may be simple to generate, they may vary according to the acquisition parameters of the diffusion weighted sequence. ADC ratios may be more time consuming to generate but, similar to our observations, many previous studies have maintained that ratios of tumor ADC to normal brain are more likely to agree with the widespread practice of visually comparing tumor signal intensity to adjacent normal brain tissue on ADC maps and DW images to determine whether diffusion is restricted, normal or facilitated in the tumor ${ }^{[2,3,18]}$. The main drawback of relying on $\mathrm{ADC}$ ratios arises from the fact that ADC of normal brain tissue decreases as the child gets older thus biasing ADC ratios, however a recent study found no association between normal brain ADC value and age $^{[22,26,27]}$.

Limitations of this study include its retrospective nature, the relatively small study cohort both in terms of the total number of lesions and the number of each histological group which may have yielded false results as regards the absence of overlap among different groups and the lack of hitopathological confirmation for DIPGs. Additionally, since ADC values are never solely relied upon in clinical practice, it may have been more valuable to assess the diagnostic performance of ADC measurements in addition to conventional MRI features in differentiation of PFTs. Furthermore, in presence of enhancement, we placed our ROIs in the enhancing areas which may have biased our results due to exclusion of non enhancing regions of the tumor. 


\section{Conclusion}

ADC values and ratios can be used to perfectly distinguish embryonal tumors and low grade gliomas. DIPGs and ependymomas exhibit intermediate ADC values between those of LGGs and EMBTs, however they can be differentiated according to location. DIPGs are pontine tumors that can be successfully differentiated from EMBTs and LGGs using ADC ratios and absolute ADC values respectively. Ependymomas are cerebellar tumors that can be successfully differentiated from EMBTs and LGGs using absolute ADC values. Larger, prospective and multicentric studies are required for validation of our results.

\section{REFERENCES}

1. Panigrahy A, Bluml S. Neuroimaging of pediatric brain tumors: from basic to advanced magnetic resonance imaging (MRI). J Child Neurol 2009; 24:1343-1365

2. Poretti A1, Meoded A, Cohen KJ, et al. Apparent diffusion coefficient of pediatric cerebellar tumors: a biomarker of tumor grade? Pediatr Blood Cancer. 2013; 60(12):2036-41.

3. Rumboldt Z, Camacho DL, Lake D, et al. Apparent diffusion coefficients for differentiation of cerebellar tumors in children. Am J Neuroradiol. 2006;27:13621369.

4. Gauvain KM, McKinstry RC, Mukherjee P, et al. Evaluating pediatric brain tumor cellularity with diffusion-tensor imaging. AJR Am

Roentgenol 2001; 177: 449- 54

5. Jaremko JL, Jans LB, Coleman LT, et al. Value and limitations of diffusion-weighted imaging in gradingand diagnosis of pediatric posterior fossa tumors. Am J Neuroradiol. 2010;31:1613-1616

6. Warren, K. E. Diffuse intrinsic pontine glioma: poised for progress. Frontiers in Oncology.2012
7. Lobel U, Sedlacik J. Reddick WE, et al. Quantitaive diffusion weighted and dynamic susceptibility weighted contrast enhanced perfusion MR imaging analysis of T2 hypointense lesion components in pediatric diffuse intrinsic glioma, American Journal of neuroradiology. 2011; 32:315322.

8. Lober RM, Cho YJ, Tang Y, et al. Diffusion-weighted MRI derived apparent diffusion coefficient identifies prognostically distinct subgroups of pediatric diffuse intrinsic pontine glioma. J Neurooncol. 2014;117:175-182.

9. Buczkowicz P, Bartels U, Bouffet E, et al. Histopathological spectrum of paediatric diffuse intrinsic pontine glioma: diagnostic and therapeutic implications. Acta Neuropathol. 2014;128(4):573-581.

10. Poretti A.,Meoded A., Huisman T. Neuroimaging of Pediatric Posterior Fossa Tumors Including Review of the Literature J MagnReson Imaging. 2012; 35:32-47.

11. Porto L., Jurcoane A., Schwabe D., et al. Differentiation between high and low grade tumours in paediatric patients by using apparent diffusion coefficients. Eur. J. Paediatr. Neurol. 2013;17:302-307

12. Murakami R., Hirai T., SugaharaT.,et al. Grading astrocytic tumors by using apparent diffusion coefficient parameters: superiority of a one- versus two-parameter pilot method. Radiology. 2009;251:838845.

13. Kono K, Inoue Y, Nakayama K, et al. The role of diffusion-weighted imaging in patients with brain tumors. AJNR Am J Neuroradiol 2001;22:1081-88

14. Zonari P., Baraldi P., Crisi G. Multimodal MRI in the characterization of glial neoplasms: the combined role of singlevoxel MR spectroscopy, diffusion imaging and echoplanar perfusion imaging. Neuroradiology. 2007;49:795-803

15. Catalaa I., Henry R., Dillon W.P., et al. Perfusion, diffusion and spectroscopy values in newly diagnosed cerebral gliomas. NMR Biomed. 2006;19:463-47. 
16. Schneider JF, Confort-Gouny S, Viola A, et al. Multiparametric differentiation of posterior fossa tumors in children using diffusion-weighted imaging and short echotime 1H-MR spectroscopy. J MagnResonImaging2007;26:1390-1398.

17. Bull JG, Saunders DE, Clark CA. Discrimination of paediatric brain tumours using apparent diffusion coefficient histograms. Eur Radiol2012;22:447-457.

18. Gimi B., Cederberg K., Derinkuyu B., et al. Utility of apparent diffusion coefficient ratios in distinguishing common pediatric cerebellar Radiol. 2012;19:794-800

19. Zitouni, S., Koc, G., Doganay, S., et al. Apparent diffusion coefficient in differentiation of pediatric posterior fossa tumors. Japanese Journal of Radiology. 2017; 35(8), 448-453

20. Koral K, Alford R, Choudhury N, et al. Applicability of apparent diffusion coefficient ratios in preoperative diagnosis of common pediatric cerebellar tumors across two institutions. Neuroradiology. 2014;56(9):781-8

21. Yeom KW, Mobley BC, Lober RM, et al. Distinctive MRI features of pediatric medulloblastoma subtypes. AJR Am J Roentgenol. 2013; 200(4):895-903.
22. Koral K, Mathis D, Gimi B, et al. Common pediatric cerebellar tumors: correlation between cell densities and apparent diffusion coefficient metrics. Radiology. 2013; 268(2):532-537

23. Fruehwald-Pallamar J, Puchner SB, Rossi $A$, et al. Magnetic resonance imaging spectrum of medulloblastoma. Neuroradiology 2011 53(6): 387-396.

24. Louis DN, Perry A, Reifenberger G et-al. The 2016 World Health Organization Classification of Tumors of the Central Nervous System: a summary. Acta Neuropathol. 2016;131 (6): 803-20

25. Song JY, Kim JH, Cho YH et-al. Treatment and outcomes for gangliogliomas: a singlecenter review of 16 patients. Brain Tumor Res Treat. 2014;2 (2): 49-55.

26. Morriss MC, Zimmerman RA, Bilaniuk LT, et al. Changes in brain water diffusion during

childhood. Neuroradiology 1999;41:929-34

27. Lovblad KO, Schneider J, Ruoss K, et al. Isotropic apparent diffusion coefficient mapping of postnatal cerebral development. Neuroradiology 2003;45:400 $-03$ 


\section{فعالية مقاييس الانتشار المشتقة من التصوير بالرنين المغناطيسي بخاصية الانتثار في التمايز بين أورام المخ الخلفية للأطفال \\ إيمان درويش ، علياء سيد شيحه ، أحمد عبد الرحمن \\ قسم الأشعة ، كلية الطب ، جامعة عين شمس ، القاهرة ، مصر}

المقدمة: نم تحديد قيمة التصوير بالرنين المغناطيسي بخاصية الانتشار (DWI) في التمايز بين أورام المخ الخام الخلفية للأطفال سابقًا ، ومع ذلك اقتصرت معظم الدراسات فقط على الأنواع الأكثر شيوعًا من أورام الميخ الخئ الخلفية وكانت نتائجها متناقضة .

الهذف من البحث:هدفت هذه الدراسة إلى تقييم دقة التصوير بالرنين المغناطيسي بخاصية الانتشار في التمييز بين اور ام المخ الخلفية للأطفال مع الحرص على تضمين أنواع أقل تو اترا ، وتوضيح النتائج القابلة للنقاش التي التي عرضت في في التين الابحاث السابقة

المرضى والطرق والحالات: قمنا بتحليل بأثز رجعي اثثان وثلاثون ورم مقسمة الي خمسة مجموعات مختلفة .

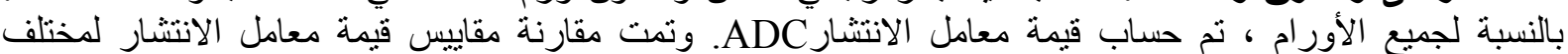

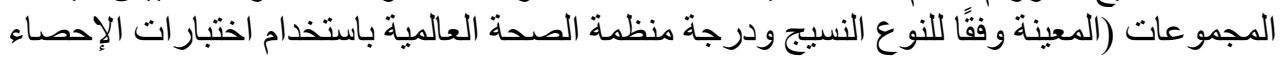

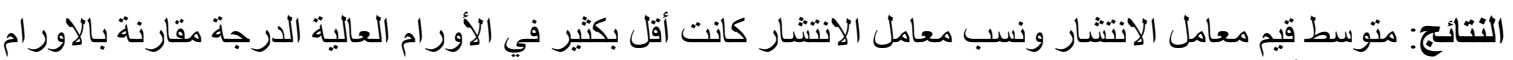

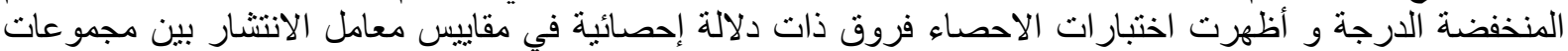

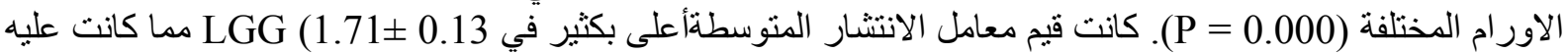

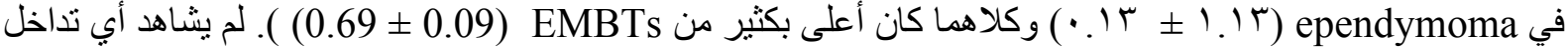

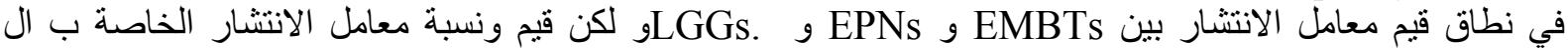
متداخلة على نطاق واسع مع EPNs والاتي لا يمكن تمييز هما باستخدام مقاييس معامل الانتشار. . . يمكن تمييز

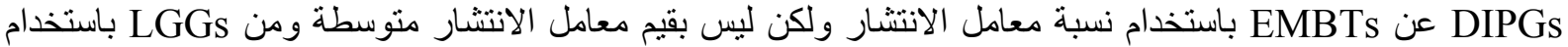

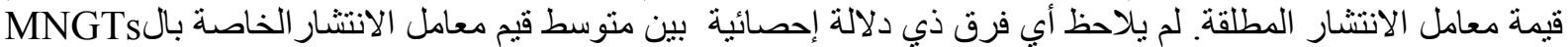

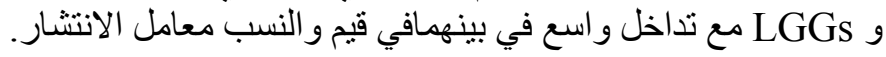
إلخاتمة: بصرف النظر عن بعض الاستثناءات ، فإن قيم ونسب معامل الانتشار .تقطع شوطًا طويلاً في تمكين التمايز

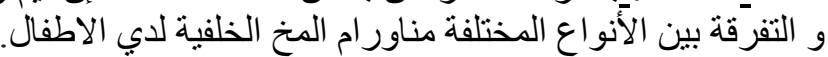

